\title{
Convergence of formal solutions of first order singular nonlinear partial differential equations in the complex domain
}

\author{
by Masatake MiYake and Akira Shirai (Nagoya)
}

Bogdan Ziemian in memoriam

\begin{abstract}
We study the convergence or divergence of formal (power series) solutions of first order nonlinear partial differential equations

$$
f\left(x, u, D_{x} u\right)=0 \quad \text { with } u(0)=0 .
$$

Here the function $f(x, u, \xi)$ is defined and holomorphic in a neighbourhood of a point $\left(0,0, \xi^{0}\right) \in \mathbb{C}_{x}^{n} \times \mathbb{C}_{u} \times \mathbb{C}_{\xi}^{n}\left(\xi^{0}=D_{x} u(0)\right)$ and $f\left(0,0, \xi^{0}\right)=0$. The equation (SE) is said to be singular if $f(0,0, \xi) \equiv 0\left(\xi \in \mathbb{C}^{n}\right)$. The criterion of convergence of a formal solution $u(x)=\sum_{|\alpha|>1} u_{\alpha} x^{\alpha}$ of (SE) is given by a generalized form of the Poincaré condition which depends on each formal solution. In the case where the formal solution diverges a precise rate of divergence or the formal Gevrey order is specified which can be interpreted in terms of the Newton polygon as in the case of linear equations but for nonlinear equations it depends on the individual formal solution.
\end{abstract}

0. Introduction. We write $x=\left(x_{1}, \ldots, x_{n}\right) \in \mathbb{C}^{n}(n \geq 1), u \in \mathbb{C}$ and $\xi=\left(\xi_{1}, \ldots, \xi_{n}\right) \in \mathbb{C}^{n}$ for the complex variables. Let $f(x, u, \xi)$ be a holomorphic function in a neighbourhood of the origin of the cited variables, and assume $f$ to be an entire function in $\xi \in \mathbb{C}^{n}$ when $x, u$ are fixed.

In this paper we are concerned with formal solutions of the first order nonlinear partial differential equation

$$
f\left(x, u(x), D_{x} u(x)\right)=0, \quad u(0)=0,
$$

where $D_{x} u=\left(D_{1} u, \ldots, D_{n} u\right)\left(D_{j}=\partial / \partial x_{j}\right)$.

We always assume that the equation (SE) is singular in the sense that

$$
f(0,0, \xi) \equiv 0, \quad \xi \in \mathbb{C}^{n}
$$

2000 Mathematics Subject Classification: 35A10, 35C10, 35F20.

Key words and phrases: singular equation, formal solution. 
We mention that this definition of singular equation is due to R. Gérard and $\mathrm{H}$. Tahara [G-T 1] where various kinds of problems for singular equations have been studied.

By the assumption $(\mathrm{S})$ the equation ( $\mathrm{SE}$ ) cannot be reduced to the normal form of Cauchy-Kowalevski type in any direction. Therefore the determination of convergence or divergence of formal solutions of (SE) is not so easy as is shown by the following example which shows the coexistence of a convergent solution and divergent solution for an equation.

EXAmple. Consider the singular ordinary differential equation

$$
f\left(x, u, u^{\prime}\right) \equiv(x-u(x)) u^{\prime}(x)-x^{2}=0, \quad u(0)=0 \quad(x \in \mathbb{C}) .
$$

Let $u(x)=\sum_{n=1}^{\infty} u_{n} x^{n}$ be a formal solution. Then by substituting this into the equation, we easily see that $\left(1-u_{1}\right) u_{1}=0$, and hence

$$
u_{1}=0 \text { or } 1 \text {. }
$$

Then we easily find that the coefficients $\left\{u_{n}\right\}_{n \geq 2}$ are uniquely determined after a choice of $u_{1}$ as above. Hence there exist just two formal solutions of the equation $(0.1)$.

For $u_{1}=0$, we set $u(x)=x v(x)$ with $v(0)=0$. Then we get the following equation for $v(x)$ :

$$
x v^{\prime}(x)+v(x)=\frac{x}{1-v(x)}, \quad v(0)=0,
$$

which is of regular singular type and $v(x)$ converges (cf. [G-T 1]).

For $u_{1}=1$, we set $u(x)=x+x^{2}+x^{2} w(x)$ with $w(0)=0$. Then we get the following equation for $w(x)$ :

$$
x^{2} w^{\prime}(x)-w=-\frac{w^{2}(x)}{1+w(x)}-2 x(1+w(x)), \quad w(0)=0,
$$

which is of irregular singular type and the formal solution $w(x)$ is divergent of Gevrey class 2 (the definition of Gevrey order will be given later).

1. Main result and related results. We always assume the existence of a formal solution of the singular equation (SE), and we write it in the form

$$
u(x)=\sum_{|\alpha| \geq 1} u_{\alpha} x^{\alpha} .
$$

We put $D_{x} u(0)=\left(\xi_{1}^{0}, \ldots, \xi_{n}^{0}\right)=\xi^{0} \in \mathbb{C}^{n}$. By the assumption (S), $f\left(0,0, \xi^{0}\right)=0$ is always satisfied, but this relation has no meaning for the formal solution. So by taking the partial derivatives in $x_{i}(i=1, \ldots, n)$ of the equation $f\left(x, u(x), D_{x} u(x)\right)=0$ we have 


$$
\begin{aligned}
\frac{\partial}{\partial x_{i}} f(x, u(x), & \left.D_{x} u(x)\right)\left.\right|_{x=0} \\
& =f_{x_{i}}\left(0,0, \xi^{0}\right)+f_{u}\left(0,0, \xi^{0}\right) \xi_{i}^{0}=0, \quad i=1, \ldots, n,
\end{aligned}
$$

because $f(0,0, \xi) \equiv 0$ by $(\mathrm{S})$. Now our result is stated as follows.

Theorem 1. (i) (Convergence criterion) Define a matrix $A\left(\xi^{0}\right)$ by

$$
A\left(\xi^{0}\right)=\left(a_{i j}\left(\xi^{0}\right)\right)_{i, j=1}^{n}:=\left(\frac{\partial^{2} f}{\partial x_{i} \xi_{j}}\left(0,0, \xi^{0}\right)+\xi_{i}^{0} \frac{\partial^{2} f}{\partial u \partial \xi_{j}}\left(0,0, \xi^{0}\right)\right)_{i, j=1}^{n}
$$

and let $\left\{\lambda_{j}\right\}_{j=1}^{n}$ be its eigenvalues. Denote by $\mathrm{Ch}\left(\lambda_{1}, \ldots, \lambda_{n}\right)$ the convex hull of $\left\{\lambda_{j}\right\}_{j=1}^{n}$ in the complex plane. Then if

$$
\mathrm{Ch}\left(\lambda_{1}, \ldots, \lambda_{n}\right) \not \supset 0 \quad \text { (Poincaré condition) }
$$

the formal solution $u(x)$ converges in a neighbourhood of the origin.

(ii) (Estimation of Gevrey order) Suppose $A\left(\xi^{0}\right)=0$ for the matrix in (1.3). Then if

$$
f_{u}\left(0,0, \xi^{0}\right) \neq 0
$$

then the formal solution $u(x)$ may diverge, and then it is of Gevrey class at most 2, which means

$$
\sum_{|\alpha| \geq 1} u_{\alpha} \frac{x^{\alpha}}{\alpha !}
$$

is convergent in a neighbourhood of the origin.

ExAmple (continued). (i) In the previous Example, $f(x, u, \xi)=x \xi-$ $u \xi-x^{2}$, and $u_{1}$ should satisfy

$$
f_{x}\left(0,0, u_{1}\right)+f_{u}\left(0,0, u_{1}\right) u_{1}=u_{1}-u_{1}^{2}=0
$$

and hence $u_{1}=0$ or 1 . We have

$$
f_{x \xi}\left(0,0, u_{1}\right)+f_{u \xi}\left(0,0, u_{1}\right) u_{1}=1-u_{1}= \begin{cases}1 & \text { if } u_{1}=0 \\ 0 & \text { if } u_{1}=1\end{cases}
$$

and $f_{u}\left(0,0, u_{1}\right)=-u_{1} \neq 0$ if $u_{1}=1$. Thus the Theorem is applicable in this case.

(ii) Under the assumptions of Theorem 1(ii), we cannot expect the convergence of formal solutions and we only give the estimation of Gevrey order from above, while we do not prove the divergence of the formal solution explicitly. Indeed, the proof of divergence is fairly difficult for general equations. Here, by the above example, we shall show that for $u_{1}=1$ the formal solution $u(x)$ is of Gevrey class 2 exactly. To do this we change the unknown function $u(x)$ to $v(x)$ by setting $u(x)=x-x v(x)(v(0)=0)$. Then we easily find the following equation for $v(x)$ :

$$
v(x)=x+v(x)^{2}+x v^{\prime}(x) v(x), \quad v(0)=0 .
$$


Let $v(x)=\sum_{n \geq 1} v_{n} x^{n}$ be the formal solution. Then we have $v_{1}=1$ and

$$
v_{n}=\sum_{i+j=n} v_{i} v_{j}+\sum_{i+j=n} i v_{i} v_{j}, \quad n \geq 2 .
$$

These imply that $v_{n}>0(n \geq 1)$ and

$$
v_{n}>(n-1) v_{n-1} v_{1}=(n-1) v_{n-1}>\ldots>(n-1) ! v_{1}=(n-1) !
$$

for $n \geq 2$. This shows that $v(x)$, and therefore $u(x)$, is a divergent series which is of Gevrey class $s$ with $s \geq 2$. (For the definition of Gevrey order $s$, see Theorem 2.) On the other hand, by the above theorem we know that $u(x)$ is of Gevrey class $s$ with $s \leq 2$. Thus we see that the formal solution $u(x)$ is of Gevrey class 2 exactly.

REMARKs. (i) In Theorem 1, if

$$
\sum_{j=1}^{n} \alpha_{j} \lambda_{j}+f_{u}\left(0,0, \xi^{0}\right) \neq 0, \quad \alpha \in \mathbb{N}^{n},|\alpha| \geq 2,
$$

then the coefficients $\left\{u_{\alpha}\right\}_{|\alpha| \geq 2}$ are uniquely determined for the formal solution.

(ii) In Theorem 1(ii), the Gevrey order will be estimated more precisely from a reduced form of the equation, and it can be interpreted in terms of a Newton polygon (cf. Theorem 2(ii) in Section 2 below).

Related results. (i) In Theorem 1(i), the Poincaré condition (Po) implies $\lambda_{j} \neq 0$ for all $j=1, \ldots, n$. But in the case where zero eigenvalues exist the problem becomes more complicated because of the existence of a nilpotent part in the Jordan canonical form of the matrix $A\left(\xi^{0}\right)$ even in linear equations. Such a case is studied by M. Hibino [ $\left.\mathrm{H}_{2}\right]$ and he characterizes the Gevrey order of divergent formal solutions for linear equations, which gives a generalization of result by T. Oshima [O]. Moreover Hibino studies the asymptotic meaning of divergent solutions from the viewpoint of Borel summability of divergent solutions for first order linear equations associated with a nilpotent vector field [H 1].

(ii) Oshima's result for linear equations in $[\mathrm{O}]$ was extended to nonlinear equations by $\mathrm{A}$. Shirai $[\mathrm{S} 1]$, who also gave a generalization of a result by Gérard and Tahara [G-T 1]. We set $t=\left(t_{1}, \ldots, t_{d}\right) \in \mathbb{C}^{d}$ and $x=$ $\left(x_{1}, \ldots, x_{m}\right) \in \mathbb{C}^{m}$, and consider the equation

$$
\begin{aligned}
\sum_{i, j=1}^{d} a_{i j}(x) t_{i} D_{t_{j}} u & +b(x) u \\
& =\sum_{j=1}^{d} c_{j}(x) t_{j}+f_{2}(x)\left(t, u,\left\{t_{i} D_{t_{j}} u\right\},\left\{D_{x_{k}} u\right\}\right)
\end{aligned}
$$


where $f_{2}(x)(\ldots)$ is a nonlinear function in the variables $\{\ldots\}$. Then under the Poincaré condition for the eigenvalues of $A(0)=\left(a_{i j}(0)\right)$, the convergence of the formal solution of the form $u(t, x)=\sum_{|\alpha| \geq 1} u_{\alpha}(x) t^{\alpha}$ (with coefficients $\left\{u_{\alpha}(x)\right\}$ holomorphic in a neighbourhood of the origin) was proved.

2. Reduction of the equation. The proof of Theorem 1 is reduced to the following,

Theorem 2. (i) (Convergence criterion) Consider the equation

$$
\sum_{j=1}^{n} \lambda_{j} x_{j} D_{j} u+b u+\sum_{j=1}^{n-1} \delta_{j} x_{j+1} D_{j} u=c_{k}(x)+g_{k+1}\left(x, u, D_{x} u\right)
$$

where $\lambda_{j}, b, \delta_{j} \in \mathbb{C}$ and

$$
\begin{gathered}
c_{k}(x)=\sum_{|\alpha|=k} c_{\alpha} x^{\alpha}, \\
g_{k+1}\left(x, u, D_{x} u\right)=\sum_{|\alpha|+k q+(k-1)|\gamma| \geq k+1} g_{\alpha q \gamma} x^{\alpha} u^{q}\left(D_{x} u\right)^{\gamma} .
\end{gathered}
$$

Assume that

$$
\operatorname{Ch}\left(\lambda_{1}, \ldots, \lambda_{n}\right) \not \supset 0
$$

and

$$
\sum_{j=1}^{n} \alpha_{j} \lambda_{j}+b \neq 0, \quad|\alpha| \geq k .
$$

Then the equation has a unique formal solution $u(x)=\sum_{|\alpha| \geq k} u_{\alpha} x^{\alpha}$ which is convergent in a neighbourhood of the origin.

(ii) (Estimation of Gevrey order) Consider the equation

$$
u(x)=c_{k}(x)+g_{k+1}\left(x, u, D_{x} u\right),
$$

with the same $c_{k}(x)$ and $g_{k+1}$ as in (2.1). Then the uniquely determined formal solution $u(x)=\sum_{|\alpha| \geq k} u_{\alpha} x^{\alpha}$ is of Gevrey class $s=1+\sigma$ with

(2.4) $\sigma=\max \left\{\frac{1}{|\alpha|+k q+(k-1)|\gamma|-k}:(\alpha, q, \gamma)\right.$ with $\left.g_{\alpha q \gamma} \neq 0, \gamma \neq 0\right\}$.

Here a formal power series $u(x)=\sum_{|\alpha| \geq k} u_{\alpha} x^{\alpha}$ is said to be of Gevrey class $s=1+\sigma$ if

$$
\sum_{|\alpha| \geq k} u_{\alpha} \frac{x^{\alpha}}{(|\alpha| !)^{\sigma}}
$$

is convergent.

REMARK (Newton polygon). The number $\sigma$ in (2.4) is interpreted in terms of the Newton polygon associated with the equation (2.3) which is 
defined as follows. First notice that by the form of the equation (2.3), we have $u(x)=O\left(|x|^{k}\right)$. To the term $x^{\alpha} u^{q}\left(D_{x} u\right)^{\gamma}$ we assign the number $|\alpha|+$ $k q+(k-1)|\gamma|$ which is the vanishing order of the term upon substituting $u(x)$ with $u(x)=O\left(|x|^{k}\right)$. In the coordinate plane $\mathbb{R}^{2}$ we define the set

$$
Q(\alpha, q, \gamma)= \begin{cases}(0,|\alpha|+k q)+Q_{2} & \text { if } \gamma=0 \\ (1,|\alpha|+k q+(k-1)|\gamma|)+Q_{2} & \text { if } \gamma \neq 0\end{cases}
$$

where $Q_{2}$ is the second quadrant $Q_{2}=\left\{(x, y) \in \mathbb{R}^{2}: x \leq 0, y \geq 0\right\}$. Now the Newton polygon $N$ associated with (2.3) is defined by

$$
N=\operatorname{Ch}\left\{Q(0,1,0) \cup Q(k, 0,0) \cup \bigcup_{(\alpha, q, \gamma)} Q(\alpha, q, \gamma)\right\},
$$

for $(\alpha, q, \gamma)$ with $g_{\alpha, q, \gamma} \neq 0$ in the expansion of $g_{k+1}$ of the equation (2.3). Here, $\mathrm{Ch}\{\ldots\}$ denotes the convex hull of points in $\{\ldots\}$, and notice that $Q(0,1,0)$ (resp. $Q(k, 0,0))$ corresponds to the term $u$ itself (resp. $\left.c_{k}(x)\right)$ in the equation (2.3). Since $Q(0,1,0)=Q(k, 0,0)$ and $|\alpha|+k q+(k-1)|\gamma| \geq k+$ 1 for $(\alpha, q, \gamma)$ with $g_{\alpha, q, \gamma} \neq 0$ by assumption, we easily see that the Newton polygon $N$ has just two vertices $(0, k)$ and $(1, k+1 / \sigma)$, and $1 / \sigma$ is the slope of the side joining the two vertices. Such a characterization of the Gevrey order of a divergent solution by the use of the Newton polygon is familiar in linear equations (cf. $[\mathrm{M}]$ and $[\mathrm{M}-\mathrm{H}]$ ). In these cases the Newton polygon is defined from the operator and does not depend on the individual formal solution which is defined as for linear singular ordinary differential equations (cf. Ramis $[\mathrm{R}]$ ). A. Shirai [S 2] studied the Gevrey order of divergent solutions of higher order nonlinear equations and determined it by using the Newton polygon defined as above. For another study of divergent formal solutions, see Gérard and Tahara [G-T 2].

Reduction procedure. The procedure of reduction to Theorem 2 is as follows. We change the unknown function $u(x)$ to $v(x)$ with $v(x)=O\left(|x|^{2}\right)$ by writing

$$
u(x)=\sum_{j=1}^{n} \xi_{j}^{0} x_{j}+\sum_{|\alpha| \geq 2} u_{\alpha} x^{\alpha}=u_{0}(x)+v(x), \quad D_{x} u(0)=\xi^{0},
$$

and take the Taylor expansion of $f\left(x, \xi^{0} \cdot x+v(x), \xi^{0}+D_{x} v\right)\left(\xi^{0} \cdot x=\right.$ $\left.\sum_{j=1}^{n} \xi_{j}^{0} x_{j}\right)$ around the point $\left(0,0, \xi^{0}\right)$. Then after a careful calculation we get an equation for $v(x)$ of the form

$$
\sum_{i, j=1}^{n} a_{i j}\left(\xi^{0}\right) x_{i} D_{j} v+b v=\sum_{|\alpha|=2} c_{\alpha} x^{\alpha}+\sum_{|\alpha|+2 q+|\gamma| \geq 3} g_{\alpha q \gamma} x^{\alpha} v^{q}\left(D_{x} v\right)^{\gamma}
$$

where $A\left(\xi^{0}\right)=\left(a_{i j}\left(\xi^{0}\right)\right)$ is given by (1.3) and $b=f_{u}\left(0,0, \xi^{0}\right)$. 
Here we notice that if $A\left(\xi^{0}\right) \neq 0$, then the condition (Po) is satisfied, and if $A\left(\xi^{0}\right)=0$ then $b \neq 0$ by the assumption (1.4).

In the case where $A\left(\xi^{0}\right)=0$, let $v(x)=O\left(|x|^{k}\right)(k \geq 2)$. Then

$$
x^{\alpha} v(x)^{q}\left(D_{x} v(x)\right)^{\gamma}=O\left(|x|^{|\alpha|+k q+(k-1)|\gamma|}\right),
$$

and for $(q, \gamma) \neq(0,0)$ we have

$|\alpha|+k q+(k-1)|\gamma|=|\alpha|+2 q+|\gamma|+(k-2)(q+|\gamma|) \geq 3+(k-2)=k+1$.

This shows that the equation is written in just the same form as in Theorem 2 (ii).

Next, let $A\left(\xi^{0}\right) \neq 0$. By making a linear transformation of independent variables so that $A\left(\xi^{0}\right)$ is reduced to the Jordan canonical form, the equation reduces to

$$
\begin{aligned}
\sum_{j=1}^{n} \lambda_{j} x_{j} D_{j} v+b v+ & \sum_{j=1}^{n-1} \delta_{j} x_{j+1} D_{j} v \\
& =\sum_{|\alpha|=2} c_{\alpha} x^{\alpha}+\sum_{|\alpha|+2 q+|\gamma| \geq 3} g_{\alpha q \gamma} x^{\alpha} v^{q}\left(D_{x} v\right)^{\gamma}
\end{aligned}
$$

where $\delta_{j}=0$ or 1 according to the Jordan canonical form.

Here we remark that we may assume $\delta_{j}$ are as small as we want by making a further change of variables $\left\{x_{j}\right\} \mapsto\left\{\varepsilon^{j} x_{j}\right\}$.

Now we take $k$ large enough so that $\sum_{j=1}^{n} \alpha_{j} \lambda_{j}+b \neq 0$ for all $\alpha \in \mathbb{N}^{n}$ with $|\alpha| \geq k$, which is possible by the Poincaré condition (Po). Then by changing the unknown function $v(x)$ to $w(x)$ below, we get the equation of the form (2.1) as we want:

$$
v(x)=\left\{\sum_{2 \leq|\alpha| \leq k-1}+\sum_{|\alpha| \geq k}\right\} u_{\alpha} x^{\alpha}=v_{0}(x)+w(x) .
$$

3. Proof of Theorem 2(i) (convergence criterion). We set

$$
P_{0}\left(x, D_{x}\right)=\sum_{j=1}^{n} \lambda_{j} x_{j} D_{j}+b+\sum_{j=1}^{n-1} \delta_{j} x_{j+1} D_{j} .
$$

Then the equation (2.1) we consider is written as

$$
P_{0}\left(x, D_{x}\right) u(x)=c_{k}(x)+g_{k+1}\left(x, u, D_{x} u\right)
$$

where $k \geq 2$ and

$$
\begin{gathered}
c_{k}(x)=\sum_{|\alpha|=k} c_{\alpha} x^{\alpha} \\
g_{k+1}\left(x, u, D_{x} u\right)=\sum_{|\alpha|+k q+(k-1)|\gamma| \geq k+1} g_{\alpha q \gamma} x^{\alpha} u^{q}\left(D_{x} u\right)^{\gamma} .
\end{gathered}
$$


The important fact is that for a homogeneous polynomial $u_{m}(x)$ of degree $m, P_{0} u_{m}(x)$ is also homogeneous of degree $m$, which means that $P_{0}\left(x, D_{x}\right)$ preserves the homogeneity. Moreover, for $m \geq k$ we have

$$
g_{k+1}\left(x, u_{m}(x), D_{x} u_{m}(x)\right)-g_{k+1}(x, 0,0)=O\left(|x|^{m+1}\right),
$$

by the condition for $g_{k+1}$.

Now we decompose the unknown function $u(x)$ into homogeneous polynomials,

(3.1) $u(x)=\sum_{m=k}^{\infty} u_{m}(x), \quad u_{m}(x)$ a homogeneous polynomial of degree $m$.

Then the above observations show the existence of a recurrence formula for $\left\{u_{m}(x)\right\}$ of the form

$$
\left\{\begin{array}{l}
P_{0}\left(x, D_{x}\right) u_{k}(x)=c_{k}(x), \\
P_{0}\left(x, D_{x}\right) u_{m}(x)=G_{m}\left(x, u_{k}(x), \ldots, u_{m-1}(x)\right), \quad m \geq k+1,
\end{array}\right.
$$

where $G_{m}(\ldots)$ are functions of $\{\ldots\}$.

We put $x \cdot D_{x}=\sum_{j=1}^{n} x_{j} D_{j}$. Then we have

$$
\left(x \cdot D_{x}\right)\left(x_{1}+\ldots+x_{n}\right)^{m}=m\left(x_{1}+\ldots+x_{n}\right)^{m},
$$

and hence

$$
\left(x \cdot D_{x}\right)^{-1}\left(x_{1}+\ldots+x_{n}\right)^{m}=\frac{1}{m}\left(x_{1}+\ldots+x_{n}\right)^{m}
$$

is well defined.

The following lemma plays a crucial role in proving the convergence of formal solutions.

LEMMA 3.1. Let $\left\{\lambda_{j}\right\}$ satisfy the conditions (Po) and (2.2). Let $H(m)$ be the set of homogeneous polynomials of degree $m$ with $m \geq k$, and consider the mapping

$$
P_{0}\left(x, D_{x}\right): H(m) \rightarrow H(m) .
$$

Then there exists a positive constant $\varepsilon$ such that if $\left|\delta_{j}\right|<\varepsilon$, then the mapping is invertible for all $m \geq k$. Moreover, an estimate $h(x) \ll H \times$ $\left(x_{1}+\ldots+x_{n}\right)^{m}(h(x) \in H(m))$ implies the estimate

$$
P_{0}^{-1} h(x) \ll C H \times \frac{\left(x_{1}+\ldots+x_{n}\right)^{m}}{m}=C H\left(x \cdot D_{x}\right)^{-1}\left(x_{1}+\ldots+x_{n}\right)^{m}
$$

with a positive constant $C$ independent of $m \geq k$. The latter relation will be denoted by

$$
P_{0}^{-1}\left(x, D_{x}\right) \ll C\left(x \cdot D_{x}\right)^{-1}
$$


Proof. We introduce a norm on $H(m)$ by

$$
\begin{aligned}
\|h\|: & =\inf \left\{H: h(x) \ll H\left(x_{1}+\ldots+x_{n}\right)^{m}\right\}, \\
& =\sup \left\{\frac{\alpha !}{m !}\left|h_{\alpha}\right|:|\alpha|=m\right\}, \quad h(x)=\sum_{|\alpha|=m} h_{\alpha} x^{\alpha} \in H(m),
\end{aligned}
$$

By the assumptions there exists a positive constant $c_{0}$ such that

$$
\left|\sum_{j=1}^{n} \alpha_{j} \lambda_{j}+b\right| \geq c_{0}|\alpha|, \quad \alpha \in \mathbb{N}^{n}(|\alpha| \geq k) .
$$

This shows that $\sum_{j=1}^{n} \lambda_{j} x_{j} D_{j}+b$ is invertible on $H(m)$ for all $m \geq k$, and we have

$$
\left\|\left(\sum_{j=1}^{n} \lambda_{j} x_{j} D_{j}+b\right)^{-1}\right\| \leq \frac{1}{c_{0} m}
$$

for the operator norm. This implies

$$
\left\|\left(\sum_{j=1}^{n} \lambda_{j} x_{j} D_{j}+b\right)^{-1}\left(\sum_{j=1}^{n-1} \delta_{j} x_{j+1} D_{j}\right)\right\| \leq \frac{n-1}{c_{0}} \max \left\{\left|\delta_{j}\right|\right\} .
$$

Hence if $\left|\delta_{j}\right|$ are so small that $\max \left|\delta_{j}\right|<c_{0} /(n-1)$, then $P_{0}\left(x, D_{x}\right)$ on $H(m)$ is invertible and we have the desired estimate (3.7) with a constant $C=1 /\left(c_{0}-(n-1) \max \left\{\left|\delta_{j}\right|\right\}\right)$.

Here we remark that the constants $\delta_{j}$ in the operator $P_{0}\left(x, D_{x}\right)$ may be assumed to be as small as we want as mentioned after (2.7). Therefore Lemma 3.1 is applicable, and the formal solution $u(x)$ of the equation $(2.1)$ is uniquely determined by solving the recursion formula (3.2).

Proof of Theorem 2(i). To prove the convergence of a formal solution we use the majorant method as follows.

First we replace the unknown function by

$$
v(x)=P_{0}\left(x, D_{x}\right) u(x), \quad \text { or } \quad u(x)=P_{0}\left(x, D_{x}\right)^{-1} v(x) .
$$

Then the equation (2.1) is equivalent to

$$
v(x)=c_{k}(x)+g_{k+1}\left(x, P_{0}^{-1} v(x), D_{x} P_{0}^{-1} v(x)\right) .
$$

We define $|x|=x_{1}+\ldots+x_{n}$, and take majorant functions of the coefficients in the equation as follows:

$$
\left\{\begin{aligned}
& c_{k}(x) \ll F|x|^{k}, \quad F>0 \\
& g_{k+1}(x, u, \xi) \ll \sum_{\alpha, q, \gamma}\left|g_{\alpha q \gamma}\right| x^{\alpha} u^{q} \xi^{\gamma}:=\left|g_{k+1}\right|(x, u, \xi) \\
& \ll \sum_{\alpha, q, \gamma}\left|g_{\alpha q \gamma}\right||x|^{|\alpha|} u^{q} \xi^{\gamma}=\left|g_{k+1}\right|(|x|, \ldots,|x|, u, \xi) .
\end{aligned}\right.
$$


Now we consider the equation

(3.11) $V(x)=F|x|^{k}+\left|g_{k+1}\right|\left(|x|, \ldots,|x|, C\left(x \cdot D_{x}\right)^{-1} V, C D_{x}\left(x \cdot D_{x}\right)^{-1} V\right)$,

where $C$ is the constant in Lemma 1. This equation has a unique formal solution of the form

$$
V(x)=\sum_{m=k}^{\infty} V_{m}\left(x_{1}+\ldots+x_{n}\right)^{m}
$$

and $V(x) \gg v(x)$ by the construction of the equation (3.11).

Here we notice the following relations:

$$
\begin{gathered}
\left(x \cdot D_{x}\right)^{-1} V(x)=\sum_{m=k}^{\infty} \frac{V_{m}}{m}\left(x_{1}+\ldots+x_{n}\right)^{m} \ll V(x), \\
D_{j}\left(x \cdot D_{x}\right)^{-1} V(x)=\sum_{m=k}^{\infty} V_{m}\left(x_{1}+\ldots+x_{n}\right)^{m-1}=\frac{V(x)}{|x|} .
\end{gathered}
$$

Now for $t \in \mathbb{C}$, we consider the following functional equation:

$$
\begin{aligned}
& W(t)=F t^{k}+\left|g_{k+1}\right|(t, \ldots, t, C W(t),\{C W(t) / t\}), \\
& W(t)=O\left(t^{k}\right) .
\end{aligned}
$$

Then we have $W\left(x_{1}+\ldots+x_{n}\right) \gg V(x)$, and therefore it is sufficient to prove the convergence of $W(t)$. Set $H(t)=W(t) / t^{k}$. Then the equation for $H(t)$ is

$$
\begin{aligned}
& H(t)=F+\frac{1}{t^{k}}\left|g_{k+1}\right|\left(t, \ldots, t, t^{k} C H(t),\left\{t^{k-1} C H(t)\right\}\right), \\
& H(0)=F .
\end{aligned}
$$

By assumption we have $g_{k+1}\left(t x, t^{k} u, t^{k-1} \xi\right)=O\left(t^{k+1}\right)\left(t x=\left(t x_{1}, \ldots, t x_{n}\right)\right)$; we see that the second term on the right hand side of (3.13) has a factor $t$, and hence it vanishes at $t=0$. Hence by the implicit function theorem there exists a unique holomorphic solution $H(t)$ in a neighbourhood of the origin.

This completes the proof of convergence of formal solutions.

4. Proof of Theorem 2(ii) (estimation of Gevrey order). Recall that the equation we consider is

$$
u(x)=c_{k}(x)+g_{k+1}\left(x, u, D_{x} u\right) .
$$

Let

$$
u(x)=\sum_{m=k}^{\infty} u_{m}(x), \quad u_{m}(x) \text { homogeneous of degree } m,
$$


be the formal solution of (2.3). Then we get the following recurrence formula for $\left\{u_{m}(x)\right\}_{m \geq k}$ :

$$
\left\{\begin{array}{l}
u_{k}(x)=c_{k}(x) \\
u_{m}(x)=\sum_{\alpha, q, \gamma} g_{\alpha q \gamma} \sum^{\prime} x^{\alpha} \prod_{i=1}^{q} u_{m_{i}}(x) \prod_{j=1}^{n} \prod_{i=1}^{\gamma_{j}} D_{j} u_{l_{j i}}(x), \quad m \geq k+1
\end{array}\right.
$$

where the summation in $\sum^{\prime}$ is over $\left\{m_{i}, l_{j i}\right\}$ satisfying

$$
|\alpha|+\sum_{i=1}^{q} m_{i}+\sum_{j=1}^{n} \sum_{i=1}^{\gamma_{j}}\left(l_{j i}-1\right)=m
$$

To find a majorant series for $u(x)$, we consider the following equation for $U(t)=\sum_{m \geq k} U_{m} t^{m}$ with $t=x_{1}+\ldots+x_{n}$ :

$$
U(t)=F t^{k}+\left|g_{k+1}\right|\left(t, \ldots, t, U(t),\left\{D_{j} U(t)\right\}\right),
$$

where the positive constant $F$ and $\left|g_{k+1}\right|$ are the same as in (3.10). Then $U\left(x_{1}+\ldots+x_{n}\right) \gg u(x)$. Therefore it is sufficient to prove the convergence of

$$
\sum_{m=k}^{\infty} U_{m} \frac{t^{m}}{(m !)^{\sigma}}
$$

with $\sigma$ defined by (2.4), that is,

$$
\sigma=\max \left\{\frac{1}{|\alpha|+k q+(k-1)|\gamma|-k}:(\alpha, q, \gamma) \text { with } g_{\alpha q \gamma} \neq 0, \gamma \neq 0\right\} .
$$

To prove this we look at the following recurrence formula for $\left\{U_{m}\right\}_{m \geq k}$ :

$$
\left\{\begin{array}{l}
U_{k}=F \\
U_{m}=\sum_{\alpha, q, \gamma}\left|g_{\alpha q \gamma}\right| \sum^{\prime} \prod_{i=1}^{q} U_{m_{i}} \times \prod_{j=1}^{n} \prod_{i=1}^{\gamma_{j}} l_{j i} U_{l_{j i}}, \quad m \geq k+1
\end{array}\right.
$$

where $\sum^{\prime}$ is as above.

We put

$$
V_{m}=U_{m} /(m !)^{\sigma}, \quad m \geq k .
$$

By dividing (4.4) by $(m !)^{\sigma}$, we get the following recurrence formula for $\left\{V_{m}\right\}$ :

$$
\left\{\begin{aligned}
V_{k}= & F /(k !)^{\sigma}, \\
V_{m}= & \sum_{\alpha, q, \gamma}\left|g_{\alpha q \gamma}\right| \sum^{\prime} \frac{\prod_{j, i} l_{j i} \times\left(\prod_{i} m_{i} ! \prod_{j, i} l_{j i} !\right)^{\sigma}}{(m !)^{\sigma}} \\
& \times \prod_{i=1}^{q} V_{m_{i}} \times \prod_{j=1}^{n} \prod_{i=1}^{\gamma_{j}} V_{l_{j i}} .
\end{aligned}\right.
$$


The crucial part in the proof is to show the inequality

$$
\frac{\prod_{j, i} l_{j i} \times\left(\prod_{i} m_{i} ! \prod_{j, i} l_{j i} !\right)^{\sigma}}{(m !)^{\sigma}} \leq A^{q+|\gamma|}
$$

with a positive constant $A$.

We postpone its proof and proceed with the proof of the Theorem. By the above inequality we have

$$
V_{m} \leq \sum_{\alpha, q, \gamma}\left|g_{\alpha q \gamma}\right| \sum^{\prime} \prod_{i=1}^{q} A V_{m_{i}} \times \prod_{j=1}^{n} \prod_{i=1}^{\gamma_{j}} A V_{l_{j i}}, \quad m \geq k+1
$$

These inequalities suggest the following functional equation for $W(t)$ to be a majorant series of $V(t)=\sum_{m \geq k} V_{m} t^{m}$ :

$$
\begin{aligned}
W(t) & =\frac{F}{(k !)^{\sigma}} t^{k}+\left|g_{k+1}\right|(t, \ldots, t, A W(t),\{A W(t) / t\}), \\
W(t) & =O\left(t^{k}\right) .
\end{aligned}
$$

Then we have $W(t) \gg V(t)$ by the construction of the above equation. Finally to prove the convergence of $W(t)$ we put

$$
Z(t)=W(t) / t^{k} .
$$

Then we get the following equation for $Z(t)$ :

$$
\begin{aligned}
& Z(t)=\frac{F}{(k !)^{\sigma}}+\frac{1}{t^{k}}\left|g_{k+1}\right|\left(t, \ldots, t, A t^{k} Z(t),\left\{A t^{k-1} Z(t)\right\}\right), \\
& Z(0)=F /(k !)^{\sigma}
\end{aligned}
$$

where the second member on the right hand side vanishes at $t=0$. Hence the implicit function theorem shows the convergence of $Z(t)$, which implies the convergence of $W(t)$ and $V(t)$ as desired.

Thus it remains to prove the inequality (4.6). In doing this, the following elementary inequality plays an important role.

Lemma 4.1. Let $m_{i} \geq k(i=1, \ldots, j)$. Then

$$
m_{1} ! \ldots m_{j} ! \leq(k !)^{j-1}\left(m_{1}+\ldots+m_{j}-(j-1) k\right) ! .
$$

The proof is by induction on $j \geq 2$, but we omit it since it is easy.

Proof of (4.6). We denote the left hand side of (4.6) by $G(\alpha, q, \gamma)$,

$$
G(\alpha, q, \gamma)=\frac{\prod_{j, i} l_{j i} \times\left(\prod_{i} m_{i} ! \prod_{j, i} l_{j i} !\right)^{\sigma}}{(m !)^{\sigma}}
$$

where

$$
m=\alpha+\sum_{i=1}^{q} m_{i}+\sum_{j=1}^{n} \sum_{i=1}^{\gamma_{j}}\left(l_{j i}-1\right)=\alpha+\sum_{i=1}^{q} m_{i}+\sum_{j=1}^{n} \sum_{i=1}^{\gamma_{j}} l_{j i}-|\gamma|,
$$


with $m_{i}, l_{j i} \geq k$. For a positive integer $L$ we have

$$
\begin{aligned}
G(\alpha, q, \gamma) & \\
\leq & \prod_{j, i} l_{j i}^{1-L \sigma} \times \frac{\left\{\prod_{i}\left(m_{i}+L\right) ! \prod_{j, i}\left(l_{j i}+L\right) !\right\}^{\sigma}}{\left(\left\{|\alpha|+\sum_{i} m_{i}+\sum_{i, j} l_{j i}-|\gamma|\right\} !\right)^{\sigma}} \\
\leq & \prod_{j, i} l_{j i}^{1-L \sigma} \times(k+L) !^{(q+|\gamma|-1) \sigma} \\
& \times \frac{\left(\left\{\sum_{i} m_{i}+\sum_{j i} l_{j i}+L(q+|\gamma|)-(q+|\gamma|-1)(k+L)\right\} !\right)^{\sigma}}{\left(\left\{|\alpha|+\sum_{i} m_{i}+\sum_{i, j} l_{j i}-|\gamma|\right\} !\right)^{\sigma}} \\
= & \prod_{j, i} l_{j i}^{1-L \sigma} \times(k+L) !^{(q+|\gamma|-1) \sigma} \\
& \times \frac{\left(\left\{\sum_{i} m_{i}+\sum_{j, i} l_{j i}-k(q+|\gamma|-1)+L\right\} !\right)^{\sigma}}{\left(\left\{|\alpha|+\sum_{i} m_{i}+\sum_{i, j} l_{j i}-|\gamma|\right\} !\right)^{\sigma}} .
\end{aligned}
$$

Here in the second inequality we applied Lemma 4.1 with $m_{i} \geq k+L$ in the lemma.

Now we divide the set of multi-indices $\{\alpha, q, \gamma\}$ with $g_{\alpha q \gamma} \neq 0$ into

$$
\begin{aligned}
& X=\{(\alpha, q, \gamma):|\alpha|+k q+(k-1)|\gamma|-k=1 / \sigma\}, \\
& Y=\{(\alpha, q, \gamma):|\alpha|+k q+(k-1)|\gamma|-k>1 / \sigma\} .
\end{aligned}
$$

We first consider the case where $(\alpha, q, \gamma) \in X$. In this case we take $L=1 / \sigma$ in (4.11). By this choice of $L$, we have $1-L \sigma=0$ and

$$
\frac{\left\{\sum_{i} m_{i}+\sum_{j, i} l_{j i}-k(q+|\gamma|-1)+L\right\} !}{\left\{|\alpha|+\sum_{i} m_{i}+\sum_{i, j} l_{j i}-|\gamma|\right\} !}=1,
$$

and hence

(4.12) $G(\alpha, q, \gamma) \leq(k+L) !^{(q+|\gamma|-1) \sigma}<(k+1 / \sigma)^{(q+|\gamma|) \sigma}, \quad(\alpha, q, \gamma) \in X$.

Next consider the case where $(\alpha, q, \gamma) \in Y$. In this case we take $L=$ $1+[1 / \sigma]$ in (4.11), where [.] denotes Gauss' symbol. By this choice of $L$ we have $l_{j i}^{1-L \sigma}<1$. On the other hand,

$$
1 / \sigma<L \leq 1 / \sigma+1<|\alpha|+k q+(k-1)|\gamma|-k+1
$$

implies $L \leq|\alpha|+k q+(k-1)|\gamma|+k$ because these are integers, and this implies

$$
\frac{\left\{\sum_{i} m_{i}+\sum_{j, i} l_{j i}-k(q+|\gamma|-1)+L\right\} !}{\left\{|\alpha|+\sum_{i} m_{i}+\sum_{i, j} l_{j i}-|\gamma|\right\} !} \leq 1 .
$$

Thus we get 


$$
\begin{aligned}
G(\alpha, q, \gamma) & \leq(k+L) !^{(q+|\gamma|-1) \sigma}<(k+1 / \sigma+1)^{(q+|\gamma|-1) \sigma} \\
& <(k+1 / \sigma+1)^{(q+|\gamma|) \sigma}, \quad(\alpha, q, \gamma) \in Y .
\end{aligned}
$$

Therefore the inequality (4.6) does hold by taking $A=(1+k+1 / \sigma)^{\sigma}$.

Thus the proof of Theorem 2(ii) is complete.

\section{References}

[G-T 1] R. Gérard and H. Tahara, Singular Nonlinear Partial Differential Equations in Complex Domain, Vieweg, 1996.

[G-T 2] - - - Formal power series solutions of nonlinear first order partial differential equations, Funkcial. Ekvac. 41 (1998), 133-166.

[H 1] M. Hibino, Gevrey asymptotic expansion for singular first order linear partial differential equations of nilpotent type, Master Thesis, Grad. School of Math., Nagoya Univ., 1998 (in Japanese).

[H 2] -, Divergence property of formal solutions for singular first order linear partial differential equations, Publ. RIMS Kyoto Univ. 35 (1999), 893-919.

[M] M. Miyake, Newton polygons and formal Gevrey indices in the Cauchy-Goursat-Fuchs type equations, J. Math. Soc. Japan 43 (1991), 305-330.

[M-H] M. Miyake and Y. Hashimoto, Newton polygons and Gevrey indices for linear partial differential operators, Nagoya Math. J. 128 (1992), 15-47.

[O] T. Oshima, On the theorem of Cauchy-Kowalevski for first order linear differential equations with degenerate principal symbols, Proc. Japan Acad. 49 (1973), 83-87.

[R] J. P. Ramis, Théorèmes d'indices Gevrey pour les équations différentielles ordinaires, Mem. Amer. Math. Soc. 48 (1984).

[S 1] A. Shirai, Convergence of formal solutions to nonlinear first order singular partial differential equations, Master Thesis, Grad. School of Math., Nagoya Univ., 1998 (in Japanese).

[S 2] - Maillet type theorem for nonlinear partial differential equations and the Newton polygons, J. Math. Soc. Japan, submitted.

Graduate School of Mathematics

Nagoya University

Nagoya, 464-8602, Japan

E-mail: mmiyake@math.nagoya-u.ac.jp 